\title{
Central aortic pressure monitoring using a brachial artery line: is it ready for use in cardiac surgery?
}

Hong Kong Med J 2018:24:321-2

DOI: $10.12809 / \mathrm{hkmj} 187299$

To the Editor-Accurate monitoring of intra-arterial pressure during low flow states is very important for patients undergoing cardiac surgery that involves cardiopulmonary bypass because inaccurate measurement of pressure can increase perioperative morbidity and mortality. Nonetheless selection of the best arterial site for pressure monitoring that is safe, uncomplicated, reliable, and accurate remains controversial.

In patients who undergo cardiac surgery, the radial, brachial, and femoral arteries are common sites for intra-arterial pressure monitoring. The radial artery is used most frequently because of its easy accessibility and the lower chance of ischaemic complications and arterial thrombosis. ${ }^{1}$ Nonetheless due to inherent problems in the measurement of central aortic pressure (eg, over- and under-estimation and radial-to-aortic pressure gradients), the use of this site in cardiac surgery that involves cardiopulmonary bypass has been criticised. ${ }^{2,3}$ Compared with that of the radial artery, femoral artery pressure more closely approximates central aortic pressure and is more reliable during cardiopulmonary bypass, but this site is associated with ischaemic and haemorrhagic complications as well as pseudoaneurysm formation. ${ }^{4}$

There is convincing evidence that measuring intra-arterial pressure using a brachial arterial site can better estimate the central aortic pressure and is more accurate and reliable than using a radial arterial site. Nonetheless until recently, concerns about the occurrence of ischaemic complications have restricted its use. The results of a new single-centre retrospective observational study of over 21000 patients have shown that using a brachial arterial line for aortic pressure monitoring during cardiac surgery is associated with fewer complications such that the combined incidence of all complications related to a brachial arterial catheter was less than $0.2 \%$ (only 41 patients). ${ }^{5}$

Nonetheless due to the biases inherent in retrospective observational studies and secondary analyses, it has not been possible to establish definitive causal relationships. Findings may have been biased due to the lack of control of confounding variables. In addition, their study ${ }^{5}$ did not compare the incidence of complications of a brachial artery catheter with those of other arterial sites such as the radial or femoral arteries. Therefore, despite their valuable findings, ${ }^{5}$ the superiority of a brachial artery approach compared with other arteries in terms of complications is unclear. A decision to use the brachial artery for central aortic pressure monitoring in low flow states such as cardiopulmonary bypass may remain equivocal.

Considering the importance of accurately measuring the haemodynamic status of a patient undergoing cardiac surgery, and the high precision of a brachial arterial catheter for central aortic pressure monitoring, as well as the low incidence of brachial catheter-related complications, this method would appear to be a better alternative to other arterial pressure monitoring methods such as radial and femoral artery lines. To inform clinical practice, further well-designed prospective comparative observational studies and clinical trials (non-inferiority of superiority trial) are warranted to determine the advantages and disadvantages of brachial artery site over other sites of intra-atrial pressure monitoring in terms of noncatheter- and catheter-related complications (either minor or major) and technically related drawbacks (eg, the accuracy of measuring).

\section{Declaration}

The author has no conflicts of interest to disclose. The author had full access to the data, contributed to the study, approved the final version for publication, and take responsibility for its accuracy and integrity.

\section{Manijeh Yousefi Moghadam *, MD}

Department of Anesthesiology and Intensive Care, School of Medicine, Sabzevar University of Medical Sciences, Sabzevar, Iran

*Corresponding author: yousefim@medsab.ac.ir

\section{References}

1. Brzezinski M, Luisetti $T$, London MJ. Radial artery cannulation: a comprehensive review of recent anatomic and physiologic investigations. Anesth Analg 2009;109:1763-81

2. Fuda G, Denault A, Deschamps A, et al. Risk factors involved in central-to-radial arterial pressure gradient during cardiac surgery. Anesth Analg 2016;122:62432.

3. Lakhal K, Robert-Edan V. Invasive monitoring of blood pressure: a radiant future for brachial artery as an alternative to radial artery catheterisation? J Thorac Dis 2017;9:4812-6.

4. Bouchard-Dechêne V, Couture P, Su A, et al. Risk factors for radial-to-femoral artery pressure gradient in patients undergoing cardiac surgery with cardiopulmonary bypass. J Cardiothorac Vasc Anesth 2018;32:692-8.

5. Singh A, Bahadorani B, Wakefield BJ, et al. Brachial arterial pressure monitoring during cardiac surgery rarely causes complications. Anesthesiology 2017;126:1065-76. 